\title{
INJECTIVITY THEOREMS IN PLANE AND SPACE
}

\author{
O. MARTIO and J. SARVAS
}

\section{Introduction}

Let $(X, d)$ be a metric space, $B$ a set and $\mathscr{M}$ some family of maps $T: A \rightarrow X$, $A \subset B$.

1.1. Proposition. Suppose that $f: X \rightarrow B$ has the following $\mathscr{M}$-approximation property: if $x_{1}, x_{2} \in X, x_{1} \neq x_{2}$, then there is $G \subset X$ and $T: f G \rightarrow X, T \in \mathscr{M}$, such that $x_{1}, x_{2} \in G$ and

$$
d(T \circ f(x), x)<d\left(x_{1}, x_{2}\right) / 2
$$

for all $x \in G$. Then $f$ is injective.

To prove this suppose that $f\left(x_{1}\right)=f\left(x_{2}\right), x_{1} \neq x_{2}$. Then

is a contradiction.

$$
\begin{aligned}
d\left(x_{1}, x_{2}\right) & \leqq d\left(T \circ f\left(x_{1}\right), x_{1}\right)+d\left(T \circ f\left(x_{2}\right), x_{2}\right) \\
& <d\left(x_{1}, x_{2}\right) / 2+d\left(x_{1}, x_{2}\right) / 2=d\left(x_{1}, x_{2}\right)
\end{aligned}
$$

In this paper we choose $X$ to be a domain $D$ in $R^{n}, n \geqq 2$, with a certain uniformity property and $\mathscr{M}$ a subclass in the set $G M(n)$ of all Möbius transformations of $\bar{R}^{n}$. It turns out that if $f: D \rightarrow R^{n}$ satisfies one of the following conditions (a)-(d), then $f$ has the $\mathscr{M}$-approximation property with respect to an appropriate family $\mathscr{M} \subset G M(n)$ and thus, by $1.1, f$ is injective :

(a) locally bi-Lipschitzian with a small Lipschitz constant,

(b) quasiregular and $n \geqq 3$ with a maximal dilatation near 1 ,

(c) analytic with a sufficiently small Schwarzian norm in $D \subset R^{2}$,

(d) an analytic function with the expression $\left|f^{\prime \prime}(z) / f^{\prime}(z)\right|$ small in $D \subset R^{2}$.

The case (a) generalizes a theorem of F. John [J], who was the first to prove a non-trivial approximation result for bi-Lipschitz mappings in terms of rigid motions. John's method was generalized by Ju. Rešetnjak [R] to cover $G M(n)$ and the approximation of type (1.2). Especially (b) is essentially based on his work.

The class (c) gives a generalization of Ahlfors' injectivity result [A], and (b) can be regarded as a counterpart of his theorem in $R^{n}, n \geqq 3$. The class (d) has been considered by Duren, Shapiro and Shields [DSS] and by Becker [B]. In the 
complex plane it is possible to use more direct methods than Rešetnjak's result to obtain $\mathscr{M}$-approximation results and hence injectivity theorems, e.g., we give a new proof to Nehari's injectivity theorem $[\mathrm{N}]$ without the best possible constant.

The paper is organized as follows: Chapter 2 is devoted to the study of so-called uniform domains, especially uniform domains in $R^{2}$ are treated in detail. In Chapter 3 the classes (a) and (b) are considered and Chapter 4 deals with (c) and (d).

Notation will be standard and generally as in [V].

\section{Uniform domains}

Two basic concepts, a John domain and a uniform domain are introduced. We prove that uniform domains are invariant under quasiconformal (qc) mappings of $R^{n}$. The last section is devoted to the study of uniform domains in $R^{2}$. It is shown that in $R^{2}$ each boundary component of a uniform domain is either a qc circle or a point and especially a Jordan domain is uniform if and only if its boundary is a qc circle.

2.1. John domains. Let $0<\alpha \leqq \beta<\infty$. A domain $D \subset R^{n}$ is called an $(\alpha, \beta)$ John domain, denoted by $D \in J(\alpha, \beta)$, if there is $x_{0} \in D$ such that every $x \in D$ has a rectifiable path $\gamma:[0, d] \rightarrow D$ with arc length as parameter such that $\gamma(0)=x$, $\gamma(d)=x_{0}$ and

$$
\begin{gathered}
d \leqq \beta, \\
\operatorname{dist}(\gamma(t), \partial D) \geqq \frac{\alpha}{d} t \text { for all } t \in[0, d] .
\end{gathered}
$$

The point $x_{0}$ is called a center of $D$. A domain $D$ is called a John domain if $D \in J(\alpha, \beta)$ for some $\alpha, \beta$. The class of all John domains in $R^{n}$ is denoted by $J$.

2.4. Remarks. (a) John domains were introduced by F. John in his study [J] of approximation of bi-Lipschitz mappings.

(b) If $D \in J(\alpha, \beta)$, then $\operatorname{diam}(D) \leqq 2 \beta$.

(c) $A$ convex domain $D$ is a John domain is and only if it is bounded; in fact, $D \in J(\alpha, \beta)$ if and only if there is $x_{0} \in D$ with $B^{n}\left(x_{0}, \alpha\right) \subset D \subset B^{n}\left(x_{0}, \beta\right)$.

2.5. A characterization of John domains. Since the image of a rectifiable path under a qc mapping need not be rectifiable, it is useful to introduce a variant of the definition which does not employ rectifiability.

Let $\mathscr{C}_{B}$ denote the class of all bounded domains $D$ in $R^{n}$ with the following property: there are $0<\delta \leqq 1$ and $x_{0} \in D$ such that for every $x \in D$ there is a path $\gamma:[0,1] \rightarrow D$ with $\gamma(0)=x, \gamma(1)=x_{0}$ and

$$
\gamma[0, t] \subset B^{n}\left(\gamma(t), \frac{1}{\delta} \operatorname{dist}(\gamma(t), \partial D)\right)
$$

for $0 \leqq t \leqq 1$. The subclass of those domains in $\mathscr{C}_{B}$ satisfying (2.6) for a given $\delta$ is denoted by $\mathscr{C}_{B}(\delta)$. 
2.7. Lemma. $J(\alpha, \beta) \subset \mathscr{C}_{B}(\alpha / \beta)$ and if $D \in \mathscr{C}_{B}(\delta)$ for some $\delta \in(0,1]$, then $D \in J\left(l / \varphi^{2}, \varphi l\right)$, where $l=\operatorname{diam}(D)$ and $\varphi \geqq 1$ depends only on $n$ and $\delta$. Thus $\mathscr{C}_{B}=J$.

Proof. Suppose that $D \in J(\alpha, \beta)$. By 2.4 (b), $D$ is bounded. Let $x_{0}$ be a center of $D$. Fix $x \in D$. Let $\gamma:[0, d] \rightarrow D$ be a path as in the definition of a John domain. Define $\gamma_{1}:[0,1] \rightarrow D$ by $\gamma_{1}(t)=\gamma(d t)$. For $0 \leqq s \leqq t \leqq 1$ we have

$$
\left|\gamma_{1}(t)-\gamma_{1}(s)\right| \leqq d(t-s) \leqq d t \leqq \frac{\beta}{\alpha} \operatorname{dist}(\gamma(d t), \partial D) \leqq \frac{\beta}{\alpha} \operatorname{dist}\left(\gamma_{1}(t), \partial D\right) .
$$

Consequently, for $\delta=\alpha / \beta$ the path $\gamma_{1}$ satisfies (2.6) and thus $D \in \mathscr{C}_{B}(\delta)$.

To prove $\mathscr{C}_{B} \subset J$ is technically more difficult. Suppose that $D \in \mathscr{C}_{B}(\delta), \delta \in(0,1]$. Fix $x \in D$. Let $\gamma:[0,1] \rightarrow D$ be a path as in 2.5 with $\gamma(0)=x \neq \gamma(1)=x_{0}$. First we construct a rectifiable path $\gamma_{1}:[0, d] \rightarrow D$ with arc length as parameter such that $\gamma_{1}(0)=x, \gamma_{1}(d)=x_{0}$ and

$$
\operatorname{dist}\left(\gamma_{1}(t), \partial D\right) \geqq 2^{-7 n} \delta^{n} t \text { for } t \in[0, d] .
$$

To simplify the construction we perform a change of parameter in $\gamma$. The mapping $f:[0,1] \rightarrow[0, a]$,

$$
f(t)=(1+t)\left(\max _{0 \leqq s \leqq t}|\gamma(s)-x|\right), \quad t \in[0,1],
$$

$a=2 \max _{0 \leqq s \leqq 1}|\gamma(s)-x|$, defines a homeomorphism since we can clearly assume $\gamma(s) \neq x$ for $s \in\left(0, \delta_{0}\right)$ for some $\delta_{0}>0$. Let $\sigma=\gamma \circ f^{-1}:[0, a] \rightarrow D$. Now by (2.5) for every $t \in[0,1]$

$$
\frac{2}{\delta} \operatorname{dist}(\gamma(t), \partial D) \geqq \max _{0 \leqq s \leqq t}|\gamma(s)-x| \geqq \frac{1}{2} f(t) .
$$

Hence the path $\sigma$ satisfies the inequalities

$$
\begin{gathered}
|\sigma(t)-x| \leqq t, \\
\varepsilon t \leqq \operatorname{dist}(\sigma(t), \partial D), \quad \varepsilon=\delta / 4
\end{gathered}
$$

for all $t \in[0, a]$.

Define a sequence $t_{0} \geqq t_{1} \geqq \ldots \geqq 0$ inductively as follows. Put $t_{0}=a$, and if $t_{i} \in[0, a]$ is defined, let

$$
t_{i+1}=\inf \left\{t \in\left[0, t_{i}\right]:\left|\sigma(t)-\sigma\left(t_{i}\right)\right| \leqq \varepsilon t_{i} / 2\right\} .
$$

Set $x_{i}=\sigma\left(t_{i}\right), i=0,1, \ldots$ Observe that $x_{i+1}$ may coincide with $x$, whence $x_{i+k}=x$ for all $k=1,2, \ldots$. By $(2.10)$ we have $B^{n}\left(x_{i}, \varepsilon t_{i}\right) \subset D$ for all $i$.

Next we show that there is an integer $\varkappa=\varkappa(n, \varepsilon)$ such that

$$
t_{i+k} \leqq \frac{1}{2} t_{i} \quad \text { for } \quad k \geqq \varkappa \quad \text { and } \quad i=0,1, \ldots
$$

Fix an integer $i$. We use a packing argument. Suppose that

$$
t_{j}>\frac{1}{2} t_{i}, \quad j=i+1, i+2, \ldots, i+k .
$$


Then $x_{j} \neq x, j=i+1, \ldots, i+k, \quad$ and $\quad x_{l} \notin B^{n}\left(x_{j}, \varepsilon t_{j} / 2\right) \quad$ whenever $i \leqq j<l \leqq i+k$. Therefore, the balls $B_{j}=B^{n}\left(x_{j}, \varepsilon t_{j} / 4\right), i<j \leqq i+k$, are disjoint, and by (2.9)

$$
B_{j} \subset B^{n}\left(x, 2 t_{i}\right) \text { for } j=i+1, \ldots, i+k .
$$

Thus if $m$ refers to the Lebesgue measure in $R^{n}$ and $\Omega_{n}=m\left(B^{n}\right)$,

$$
\Omega_{n}\left(2 t_{i}\right)^{n}=m\left(B^{n}\left(x, 2 t_{i}\right)\right) \geqq \sum_{j=i+1}^{i+k} m\left(B_{j}\right)>k \Omega_{n}\left(\varepsilon t_{i} / 8\right)^{n} .
$$

This yields $k<2^{4 n} \varepsilon^{-n}$. We now obtain (2.11) by choosing the integer $x$ in such a way that

$$
0 \leqq x-2^{4 n} \varepsilon^{-n}<1 .
$$

Furthermore, (2.11) implies that $t_{i} \backslash 0$ and $x_{i} \rightarrow x$ as $i \rightarrow \infty$.

Define a path $\gamma_{2}:[0, d] \rightarrow D$ as the broken line joining $x_{i}$ to $x_{i+1}, i=0,1, \ldots$, with arc length as parameter. We show that $d<\infty$, i.e., $\gamma_{2}$ is rectifiable, and that $\gamma_{1}(t)=\gamma_{2}(d-t), t \in[0, d]$, is the required path in (2.8).

Fix $t \in[0, d)$ and set $y=\gamma_{2}(t)$. Then $y$ lies on the line segment joining $x_{i_{0}}$ and $x_{i_{0}+1}$ for some $i_{0}$. Let $l_{y} \leqq \infty$ denote the length of $\gamma_{2} \mid[t, d)$. We have by $(2.11)$

$$
l_{y} \leqq \sum_{j=i_{0}}^{\infty}\left|x_{j+1}-x_{j}\right|=\sum_{j=i_{0}}^{\infty} \varepsilon t_{j} / 2=\frac{1}{2} \varepsilon \sum_{k=0}^{\infty}\left(\sum_{j=k \varkappa}^{(k+1) \varkappa-1} t_{i_{0}+j}\right) \leqq \frac{1}{2} \varepsilon \sum_{k=0}^{\infty} x 2^{-k} t_{i_{0}}=\varepsilon \varkappa t_{i_{0}} .
$$

On the other hand, dist $(y, \partial D) \leqq \varepsilon t_{i_{0}} / 2$ by (2.10) since $x_{i_{0}+1} \in \bar{B}^{n}\left(x_{i_{0}}, \varepsilon t_{i_{0}} / 2\right)$. Because $t_{i_{0}}>0$, we get

$$
\frac{\operatorname{dist}(y, \partial D)}{l_{y}} \geqq \frac{\varepsilon t_{i_{0}} / 2}{\varepsilon \varkappa t_{i_{0}}}=\frac{1}{2 \varkappa} \geqq \frac{1}{2\left(2^{4 n} \varepsilon^{-n}+1\right)} \geqq 2^{-5 n} \varepsilon^{n} .
$$

Thus $l_{y} \leqq \varepsilon x t_{i_{0}} \leqq \varepsilon x t_{0}<\infty$, and since $2^{-5 n} \varepsilon^{n}=2^{-7 n} \delta^{n}, \gamma_{1}$ is the required path.

To complete the proof we observe that since $D \in \mathscr{C}_{B}$, the domain $D$ is bounded and, consequently, in the above construction

$$
a=2 \max _{0 \leqq s \leqq 1}|\gamma(s)-x| \leqq 2 \operatorname{diam}(D) .
$$

Thus if we let $l=\operatorname{diam}(D)$,

$$
d \leqq \varepsilon x t_{0}=\varepsilon \varkappa a \leqq \frac{\delta}{4}\left(1+2^{4 n} \varepsilon^{-n}\right) a \leqq 2^{6 n} \delta^{-n} l .
$$

On the other hand, the property $D \in \mathscr{C}_{B}(\delta)$ implies that $B=B^{n}\left(x_{0}, \delta l / 2\right) \subset D$.

Let now $x \in D$ be arbitrary. We may assume that $x \notin B$. Let $\gamma_{1}:[0, d] \rightarrow D$ be the path constructed above. By (2.8)

$$
\operatorname{dist}\left(\gamma_{1}(t), \partial D\right) \geqq 2^{--7 n} \delta^{n} t \geqq 2^{-7 n} \delta^{n} d t / d \geqq 2^{-8 n} \delta^{n+1} l t / d ;
$$

thus for $\alpha=2^{-8 n} \delta^{n+1} l, \beta=2^{6 n} \delta^{-n} l$ the domain $D$ is in $J(\alpha, \beta)$. If we set $\varphi=2^{6 n} \delta^{-n}$ the proof is complete. 
2.12. Uniform domains. Let $0<\alpha \leqq \beta<\infty$. A domain $D \subset R^{n}$ is called $(\alpha, \beta)$ uniform if for each pair $x, y$ of points in $D, x \neq y$, there is $G \in J(\alpha|x-y|, \beta|x-y|)$ such that $x, y \in G \subset D$. The collection of all $(\alpha, \beta)$-uniform domains is denoted by $U(\alpha, \beta)$. A domain $D \subset R^{n}$ is called uniform, and written as $D \in U$, if $D \in U(\alpha, \beta)$ for some $\alpha, \beta$.

2.13. Examples and remarks. (a) It is not difficult to see that $B^{n}(x, r) \in U(1 / 2, \sqrt{2} / 2)$ and $R^{n} \in U(1 / 2, \varepsilon+1 / 2)$ for every $\varepsilon>0$.

(b) A John domain need not be uniform, e.g.,

$$
D=B^{n} \backslash\left\{x \in R^{n}: x_{n}=0, x_{1} \geqq 0\right\}
$$

is a John domain but not uniform.

(c) Properties of convex sets imply: If $D \in J$ is convex, then $D \in U$.

2.14. Quasiconformal invariance of uniform domain. Let $D \subset R^{n}$ be a domain and $f: D \rightarrow R^{n}$ a bi-Lipschitz mapping, i.e., for some $L \geqq 1$

$$
|x-y| / L \leqq|f(x)-f(y)| \leqq L|x-y|
$$

for all $x, y \in D$. It is easy to see that if $D \in J(\alpha, \beta)$, then $f D \in J\left(L^{-3} \alpha, L \beta\right)$. This implies that if $D$ is uniform, $f D$ is also uniform. However, simple examples show that if $f: D \rightarrow R^{n}$ is qc and $D$ uniform, then $f D$ need not be uniform. For instance, take $D=B^{n}$ and map $B^{n}$ quasiconformally onto a domain $D^{\prime}$ which has an outward directed spire (see [GV, p. 56]) and it is easy to see that $D^{\prime}$ is not uniform. For $n=2$ the Koebe mapping $f(z)=z /(1-z)^{2}$ of $B^{2}$ gives a well-known example. The next theorem shows that the situation is different if we consider qc mappings of the whole $R^{n}$.

2.15. The or em. Suppose that $f: R^{n} \rightarrow R^{n}, n \geqq 2$, is a K-qc mapping and $D \in U(\alpha, \beta)$. Then $f D \in U\left(\varepsilon^{2}, 1 / \varepsilon\right)$, where $\varepsilon \in(0,1]$ depends only on $n, K, \alpha$ and $\beta$.

For the proof we need a fairly standard distortion argument.

2.16. Lemma. Let $f$ be as above and

$$
l(x, f, r)=\inf _{|y-x|=r}|f(y)-f(x)|, \quad L(x, f, r)=\sup _{|y-x|=r}|f(y)-f(x)| .
$$

If $0<r_{1} \leqq r_{2}<\infty$, then

$$
L\left(x, f, r_{2}\right) / l\left(x, f, r_{1}\right) \leqq c\left(r_{2} / r_{1}\right)^{K^{1 /(n-1)}},
$$

where $c<\infty$ depends only on $n$ and $K$.

Proof. First, reasoning as in [V, p. 79] we get

$$
L(x, f, r) / l(x, f, r) \leqq c^{\prime}=c^{\prime}(n, K) .
$$

Now, let $0<r_{1} \leqq r_{2}<\infty$. By (2.18)

$$
L\left(x, f, r_{2}\right) / l\left(x, f, r_{1}\right) \leqq c^{\prime 2} l\left(x, f, r_{2}\right) / L\left(x, f, r_{1}\right),
$$


and we may assume $l\left(x, f, r_{2}\right)>L\left(x, f, r_{1}\right)$. Let $\Gamma$ be the family of all paths joining $S^{n-1}\left(x, r_{1}\right)$ and $S^{n-1}\left(x, r_{2}\right)$ in $B^{n}\left(x, r_{2}\right)$ and $M(\Gamma)$ its $n$-modulus. Then

$$
\omega_{n-1}\left(\log \left(l\left(x, f, r_{2}\right) / L\left(x, f, r_{1}\right)\right)\right)^{1-n} \geqq M(f \Gamma) \geqq \frac{1}{K} M(\Gamma)=\frac{\omega_{n-1}}{K}\left(\log \left(r_{2} / r_{1}\right)\right)^{1-n},
$$

where $\omega_{n-1}$ is the $(n-1)$-measure of $S^{n-1}$. This combined with (2.19) gives (2.17).

2.20. Proof of Theorem 2.15. Suppose that $D \in U(\alpha, \beta)$ and let $x^{\prime}, y^{\prime} \in f D, x^{\prime} \neq y^{\prime}$. Put $x=f^{-1}\left(x^{\prime}\right)$ and $y=f^{-1}\left(y^{\prime}\right)$. Now there is $G \in J(\alpha|x-y|, \beta|x-y|)$ such that $x, y \in G \subset D$. We show that $f G \in J\left(\varepsilon^{2}\left|x^{\prime}-y^{\prime}\right|,\left|x^{\prime}-y^{\prime}\right| / \varepsilon\right)$, where $\varepsilon \in(0,1]$ depends only on $n, K, \alpha$ and $\beta$.

With $\delta=\alpha / \beta$ Lemma 2.7 gives $G \in \mathscr{C}_{B}(\delta)$. We prove that $f G \in \mathscr{C}_{B}\left(c \delta^{K^{\prime}}\right)$, where $K^{\prime}=K^{1 /(n-1)}$ and $c \in(0,1]$ depends only on $n$ and $K$.

Let $x_{0}, \delta$ and $\gamma$ be as in (2.6) when $D$ is replaced by $G$. It is sufficient to show that

$$
(f \circ \gamma)[0, s] \subset B^{n}\left(f \circ \gamma(s), \frac{1}{\delta^{\prime}}, \operatorname{dist}(f \circ \gamma(s), \partial G)\right)
$$

for every $s \in[0,1]$ with $\delta^{\prime}=c \delta^{K^{\prime}}$. Fix $s \in[0,1]$. Set $d=\operatorname{dist}(\gamma(s), \partial G)$. Let $l_{1}=$ $l(\gamma(s), f, d), L_{2}=L(\gamma(s), f, d / \delta)$; for notation see Lemma 2.16. Because $B^{n}(\gamma(s), d) \subset G$ and $\gamma[0, s] \subset B^{n}(\gamma(s), d / \delta)$ we get $B^{n}\left(f \circ \gamma(s), l_{1}\right) \subset f G$, and thus $l_{1} \leqq \operatorname{dist}(f \circ \gamma(s), \partial f G)$, and

$$
(f \circ \gamma)[0, s] \subset B^{n}\left(f \circ \gamma(s), L_{2}\right) .
$$

To obtain (2.21) we only need to show $L_{2} \leqq l_{1} / \delta^{\prime}$. But this follows from Lemma 2.16. Thus $f G \in \mathscr{C}_{B}\left(\delta^{\prime}\right)$.

In view of the second claim in Lemma 2.7 it suffices to find an upper bound for $\operatorname{diam}(f G)$ in terms of $\left|x^{\prime}-y^{\prime}\right|$. Since $G \in J(\alpha|x-y|, \beta|x-y|)$, diam $G \leqq 2|x-y| \beta$ by (2.4) (b). On the other hand, by Lemma 2.16,

$$
\operatorname{diam}(f G) \leqq 2 L(x, f, 2|x-y| \beta) \leqq 2 c l(x, f,|x-y|) \leqq 2 c\left|x^{\prime}-y^{\prime}\right|,
$$

where $c$ depends on $n, K$ and $\beta$. This completes the proof.

2.22. Remark. The proof for Theorem 2.15 shows that if $f: R^{n} \rightarrow R^{n}$ is qc and $D \subset R^{n}$ a John domain, then also $f D$ is a John domain.

2.23. Uniform domains in $R^{2}$. A Jordan curve $C$ in $\bar{R}^{2}$ is called a qc circle if there is a qc mapping $f: \bar{R}^{2} \rightarrow \bar{R}^{2}$ with $f S^{1}=C$. Here we show, among other things, that a Jordan domain in $R^{2}$ is uniform if and only if its boundary is a qc circle. This gives a new characterization of qc circles.

2.24. Theorem. Suppose that $D \subset R^{2}$ is a uniform domain. Then each boundary component of $D$ in $\bar{R}^{2}$ is either a point or a qc circle in $\bar{R}^{2}$.

To prove Theorem 2.24 we first introduce a simple metric condition which is sufficient to make a Jordan curve a qc circle.

A closed set $A \subset \bar{R}^{2}$ is said to be of $q$-bounded distortion, $0<q \leqq 1$, if for all $x \in A \cap R^{2}$ and $r>0$ the $\operatorname{disc} B^{2}(x, q r)$ meets only the $x$-component of $A \cap \bar{B}^{2}(x, r)$. 
2.25. Lemma. If a Jordan curve $C \subset \bar{R}^{2}$ is of q-bounded distortion, then it is a qc circle.

Proof. Suppose first that $\infty \in C$. Let $z_{1}, z_{2}, z_{3} \in R^{2}$ be three points on $C$ in this order. If $q\left|z_{1}-z_{2}\right|>\left|z_{1}-z_{3}\right|$, then clearly $C$ cannot have $q$-bounded distortion. Consequently,

$$
\left|z_{1}-z_{2}\right| /\left|z_{1}-z_{3}\right| \leqq 1 / q .
$$

This is Ahlfors' condition in [A, Theorem 1], which shows that $C$ is a qc circle.

Next, suppose that $C \subset R^{2}$. We may assume $0<q \leqq 1 / 2$. Again, by Ahlfors' criterion [A, p. 295], $C$ is a qc circle if

$$
\frac{\left|z_{1}-z_{2}\right|}{\left|z_{1}-z_{3}\right|} \frac{\left|z_{3}-z_{4}\right|}{\left|z_{2}-z_{4}\right|} \leqq c
$$

for arbitrary four points $z_{i} \in C$ such that $z_{2}$ and $z_{4}$ belong to different components of $C \backslash\left\{z_{1}, z_{3}\right\}$. We show that (2.26) holds for $c=q^{-4}$.

Let $z_{i}, \quad i=1,2,3,4$, be four points on $C$ as above. Set $\alpha=\left|z_{1}-z_{2}\right| /\left|z_{1}-z_{3}\right|$. Suppose that $\alpha>1 / q^{2}$. Now $\left|z_{1}-z_{4}\right| \leqq\left|z_{1}-z_{3}\right| / q$ since otherwise $C$ cannot be of $q$-bounded distortion. But this gives

$$
\left|z_{1}-z_{4}\right| \leqq\left|z_{1}-z_{3}\right| / q=\left|z_{1}-z_{2}\right| /(\alpha q)<q\left|z_{1}-z_{2}\right| .
$$

On the other hand, if we set $t=\left|z_{1}-z_{2}\right| /\left|z_{2}-z_{4}\right|$ the inequality (2.27) yields

$$
t \leqq \frac{\left|z_{1}-z_{4}\right|+\left|z_{4}-z_{2}\right|}{\left|z_{2}-z_{4}\right|} \leqq \frac{q\left|z_{1}-z_{2}\right|+\left|z_{4}-z_{2}\right|}{\left|z_{2}-z_{4}\right|}=t q+1 .
$$

This gives $t \leqq 1 /(1-q) \leqq 2$. Together with

$$
\left|z_{3}-z_{4}\right| \leqq\left|z_{3}-z_{1}\right|+\left|z_{1}-z_{4}\right| \leqq\left(1+\frac{1}{q}\right)\left|z_{3}-z_{1}\right| \leqq \frac{2}{q}\left|z_{1}-z_{3}\right|
$$

the inequality $t \leqq 2$ implies (2.26) with $c=4 / q<1 / q^{4}$.

If $\beta=\left|z_{3}-z_{4}\right| /\left|z_{2}-z_{4}\right|>1 / q^{2}$, then the same reasoning gives $c<1 / q^{4}$ in (2.26). Finally, if $\alpha, \beta \leqq 1 / q^{2}$, then (2.26) holds with $c=1 / q^{4}$. This completes the proof.

2.28. Remark. Lemma 2.25 and the proof for Theorem 2.24 together with Corollary 2.32 below show that a Jordan curve in $\bar{R}^{2}$ is a qc circle if and only if it is of $q$-bounded distortion for some $q \in(0,1]$.

2.29. Lemma. If $D \subset R^{2}$ is a uniform domain, then each boundary component of $D$ in $\bar{R}^{2}$ is either a point or a Jordan curve.

Proof. It is well-known (see e.g. [Ne, Theorem 16.3, p. 168]) that it suffices to show that $D$ is locally connected at each boundary point $x \in \bar{R}^{2}$. This means that each neighborhood $U$ of $x$ contains a neighborhood $V$ of $x$ such that every pair of points $x_{1}, x_{2}$ in $V \cap D$ can be joined in $U \cap D$. Let $D \in U(\alpha, \beta)$. We may assume $\beta \geqq 1$. 
Suppose first that $x \in \partial D \cap R^{2}$. Let $U$ be a neighborhood of $x$. Pick $r>0$ in such a way that $B^{2}(x, r) \subset U$. Set $V=B^{2}(x, r /(4 \beta+1))$. Fix $x_{1}, x_{2} \in V \cap D$. Since $D \in U(\alpha, \beta)$, there is $G \in J\left(\alpha\left|x_{1}-x_{2}\right|, \beta\left|x_{1}-x_{2}\right|\right)$ with $x_{1}, x_{2} \in G \subset D$. Now diam $G \leqq$ $2 \beta\left|x_{1}-x_{2}\right|$. Hence for every $y \in G$

$$
|y-x| \leqq\left|y-x_{1}\right|+\left|x_{1}-x\right|<2 \beta\left|x_{1}-x_{2}\right|+\frac{r}{4 \beta+1}<\frac{2 \beta 2 r}{4 \beta+1}+\frac{r}{4 \beta+1}=r
$$

and so $G \subset U \cap D$. Thus the points $x_{1}, x_{2}$ can be joined in $U \cap D$.

Suppose that $x=\infty \in \partial D$ and let $U$ be a neighborhood of $\infty$. Fix $r>0$-in such a way that $\bar{R}^{2} \backslash \bar{B}^{2}(r) \subset U$. Set $V=\bar{R}^{2} \backslash \bar{B}^{2}(4 r \beta / \alpha)$. Let $x_{1}, x_{2} \in V \cap D$. Pick $G$ as above. Then $x_{1}$ and $x_{2}$ can be joined in $G$ by the composed path $\gamma_{2}^{-1} \gamma_{1}$, where $\gamma_{i}$ is the path joining $x_{i}, i=1,2$, to the center of $G$. If $\gamma_{i}$ meets $\bar{B}^{2}(r)$ at a point $y_{i}=$ $\gamma_{i}\left(t_{i}\right), i=1$ or 2 , then

$$
\begin{aligned}
\operatorname{dist}\left(y_{i}, \partial D\right) & \geqq \frac{\alpha}{\beta} t_{i} \geqq \frac{\alpha}{\beta}\left|x_{i}-y_{i}\right| \geqq \frac{\alpha}{\beta}\left(\left|x_{i}\right|-\left|y_{i}\right|\right) \geqq \frac{\alpha}{\beta}(4 r \beta / \alpha-r) \\
& \geqq \frac{\alpha}{\beta}(4 r \beta / \alpha-\beta r / \alpha) \geqq 3 r .
\end{aligned}
$$

2.30. Remark. It is not difficult to show that for $n=2$ a uniform domain is always $b$-locally connected; see [G, p. 567]. Thus [G, Lemma 5] gives the same result as Theorem 2.24 .

2.31. Proof of Theorem 2.24. Suppose that $D \in U(\alpha, \beta)$ and let $C \subset \bar{R}^{2}$ be a boundary component of $D$. Then by Lemma 2.29 it is either a point or a Jordan curve. Thus if $C$ is a Jordan curve it suffices to show, in view of Lemma 2.25, that $C$ is of $q$-bounded distortion for some $q \in(0,1]$.

Let $q=\min (\alpha /(\alpha+\beta), 1 /(1+2 \beta))$. Suppose that $C$ is not of $q$-bounded distortion. Then there is $x \in C$ and $r>0$ such that $B^{2}(x, q r)$ meets a component $K_{1}$ of $C \cap \bar{B}, B=B^{2}(x, r)$, which is not the $x$-component $K_{2}$ of $C \cap \bar{B}$. Let $U_{i}$ be the component of $B \cap D$ which contains $K_{i}$ as a part of a boundary, $i=1,2$. There are two possibilities: (a) $U_{1}=U_{2}$. Now it is easy to see that there are points $x_{1}, x_{2} \in D \backslash B^{2}(x, r)$ which can be joined in $D$ only through $B^{2}(x, q r)$. (b) $U_{1} \neq U_{2}$. Pick $x_{i} \in B^{2}(x, q r) \cap U_{i}, i=1,2$. If $x_{1}$ and $x_{2}$ are joined by a path $\gamma$ in $D$, then clearly $\gamma$ meets $\left\lceil B^{2}(x, r)\right.$.

In both cases (a) and (b) there is a domain $G \in J\left(\alpha\left|x_{1}-x_{2}\right|, \beta\left|x_{1}-x_{2}\right|\right)$ with $x_{1}, x_{2} \in G \subset D$. Let $\gamma_{i}:\left[0, d_{i}\right] \rightarrow G$ be the paths joining $x_{i}$ to the center $x_{0}$ of $G$. In the case (a) one of the paths, say $\gamma_{1}$, meets $B^{2}(x, q r)$. Let $\gamma_{1}(s) \in B^{2}(x, q r)$. Then $s>r(1-q)$ and since $\beta\left|x_{1}-x_{2}\right| \geqq d_{1}$ we have

$$
\frac{\alpha}{\beta} r(1-q)=\frac{\alpha}{\beta} \frac{\left|x_{1}-x_{2}\right|}{\left|x_{1}-x_{2}\right|} r(1-q)<\frac{\alpha\left|x_{1}-x_{2}\right|}{d_{1}} s \leqq \operatorname{dist}\left(\gamma_{1}(s), \partial D\right) \leqq q r,
$$

which yields $\alpha(1-q) / \beta<q$. This is a contradiction since $q \leqq \alpha /(\alpha+\beta)$. In the case (b), $d_{i} \geqq r(1-q), i=1$ or 2 . Thus

$$
r(1-q) \leqq d_{i} \leqq \beta\left|x_{1}-x_{2}\right|<2 \beta r q,
$$

which gives $1-q<2 \beta q$ contradicting $q \leqq 1 /(1+2 \beta)$. The proof is complete. 
2.32. Remarks. (a) The proof of Theorem 2.24 shows that every boundary component of a domain $D \in U(\alpha, \beta)$ in $R^{2}$ is of $q$-bounded distortion, where $q$ depends only on $\alpha$ and $\beta$.

(b) Theorem 2.24 should be compared with a theorem of Gehring [G, Theorem 6]: Let $D \subset R^{2}$ be a domain with the following property. There is $\delta>0$ such that $\sup _{z \in D}\left|S_{f}(z)\right|$ dist $(z, \partial D)^{2}<\delta$ implies that $f$ is injective in $D$ whenever $f$ is analytic in $D$. Here $S_{f}$ refers to the Schwarzian derivative of $f$ in $D$. Then every boundary component of $D$ is either a point or a $K$-qc circle, $K$ depending only on $\delta$. This result with Theorem 4.24 of Chapter 4 gives an alternative proof for Theorem 2.24.

Theorems 2.15 and 2.24 yield

2.33. Corollary. Let $D \subset R^{2}$ be a Jordan domain in $\bar{R}^{2}$. Then $D$ is uniform if and only if $\partial D$ is a qc circle.

\section{Approximation and injectivity theorems in $R^{n}$}

An "inverse mapping" approximation theorem due to Ju. Rešetnjak is presented. Proposition 1.1 is then applied to obtain injectivity theorems for locally bi-Lipschitz mappings in $R^{n}, n \geqq 2$, and for quasiregular mappings in $R^{n}, n \geqq 3$.

3.1. Approximation theorem of Rešetnjak. Let $G M(n)$ be the set of all Möbius transformations $T: \bar{R}^{n} \rightarrow \bar{R}^{n}$ and let $\mathscr{M}$ be a subset of $G M(n)$. Suppose that $f: D \rightarrow R^{n}$, $D \subset R^{n}$ a domain, is a continuous mapping with the following property: there are numbers $\lambda \geqq 0$ and $0<q \leqq 1$ such that for each $B^{n}(y, r) \subset D$ there exists $L \in \mathscr{M}$ with

$$
|L \circ f(x)-x| \leqq \lambda r \text { for } x \in B^{n}(y, q r) .
$$

We then say that $f$ has an $\mathscr{M}(q, \lambda)$-approximation property.

The theorem of Rešetnjak [R, Basic Lemma] can now be rephrased as follows.

3.3. Theorem. Suppose that $D \in J(\alpha, \beta)$ and $0<q \leqq 1$. There is $\lambda_{0}>0$ depending only on $\alpha / \beta$ and $q$ such that if $0<\lambda \leqq \lambda_{0}$ and if $f: D \rightarrow R^{n}$ has the $\mathscr{M}(q, \lambda)$ approximation property, then there exists $T \in \mathscr{M}$ with

$$
|T \circ f(x)-x| \leqq \mu_{0} \beta \lambda
$$

for all $x \in D$ and $\mu_{0}$ depends only on $\alpha / \beta$ and $q$.

3.5. Remark. The proof of [R, Basic Lemma] can be used to show

$$
\lambda_{0}=\frac{q^{3}}{5400}\left(\frac{\alpha}{\beta}\right)^{2}, \quad \mu_{0}=\frac{1400}{q^{3}}\left(\frac{\beta}{\alpha}\right)^{2} .
$$

3.6. Locally bi-Lipschitzian mappings. A mapping $f: G \rightarrow R^{n}, G$ a domain in $R^{n}, n \geqq 2$, is a locally L-bi-Lipschitz mapping if for every $x_{0} \in G$ and $L^{\prime}>L$ there is $B^{n}\left(x_{0}, r\right) \subset G$ such that

$$
1 / L^{\prime} \leqq|f(x)-f(y)| /|x-y| \leqq L^{\prime}
$$

for all $x, y \in B^{n}\left(x_{0}, r\right), x \neq y$. 
F. John [J] has proved:

3.7. Theorem. Let $f: B^{n}\left(x_{0}, r\right) \rightarrow R^{n}$ be a locally L-bi-Lipschitz mapping $1 \leqq$ $L<2$. Then there exist a rigid motion $T: R^{n} \rightarrow R^{n}$ such that

$$
|T \circ f(x)-x| \leqq c_{n} r(L-1) \text { for }\left|x-x_{0}\right| \leqq q r,
$$

where $q=\left(1+(L-1)^{1 / 2} /(2-L)\right)^{-1}$ and $c_{n}$ depends only on $n \geqq 2$.

Theorem 3.3 can now be used to prove global approximation results in John domains. In fact, a theorem of this type was proved by F. John; see [J, Theorem II]. However, he stated the approximation in the form $|f(x)-T(x)| \leqq \mu \lambda$, which makes no difference to the form of Theorem 3.7 if $T$ is a rigid motion.

In the next theorem we combine Theorems 3.3, 3.7 and Proposition 1.1.

3.8. Theorem. Let $G \subset R^{n}, n \geqq 2$, be an $(\alpha, \beta)$-uniform domain and $f: G \rightarrow R^{n}$ a locally L-bi-Lipschitz mapping. There is $c>0$ depending only on $n, \alpha$ and $\beta$ such that $f$ is injective in $G$ whenever $1 \leqq L \leqq 1+c$.

Proof. Let $\mathscr{M}$ be the set of all rigid motions $T: R^{n} \rightarrow R^{n}, n \geqq 2$. Suppose that $G \in U(\alpha, \beta)$. Write $q=(1+\sqrt{2})^{-1}$. Let $\lambda_{0}>0$ and $\mu_{0}>0$ be the constants of Theorem 3.3 depending on $n, \alpha / \beta$ and $q$ defined above. Let $c=\min \left(1 / 2,\left(4 \mu_{0} \beta c_{n}\right)^{-1}, \lambda_{0} / c_{n}\right)$, where $c_{n}$ is the constant of Theorem 3.7.

Suppose that $f: G \rightarrow R^{n}$ is locally L-bi-Lipschitzian with $1 \leqq L \leqq 1+c$. We show that $f$ satisfies the condition (1.2). Proposition (1.1) then implies that $f$ is injective.

First we observe that $f$ satisfies the $\mathscr{M}(q, \lambda)$-approximation property (3.2) with $\lambda=c_{n} c$. This follows from Theorem 3.7 since

$$
|T \circ f(x)-x| \leqq c_{n}(L-1) r \leqq c_{n} c r=\lambda r
$$

for $\left|x-x_{0}\right| \leqq q r$ whenever $B^{n}\left(x_{0}, r\right) \subset G$.

Suppose now that $x_{1}, x_{2} \in G, x_{1} \neq x_{2}$. Since $G$ is $(\alpha, \beta)$-uniform, there is $D \in J\left(\alpha\left|x_{1}-x_{2}\right|, \beta\left|x_{1}-x_{2}\right|\right)$ with $x_{1}, x_{2} \in D \subset G$. The mapping $f \mid D$ has the $\mathscr{M}(q, \lambda)$ approximation property for $\lambda \leqq \lambda_{0}$; hence, by Theorem 3.3, there exists $T \in \mathscr{M}$ with

$$
|T \circ f(x)-x| \leqq \mu_{0} \lambda \beta\left|x_{1}-x_{2}\right|=\mu_{0} \beta c_{n} c\left|x_{1}-x_{2}\right| \leqq \frac{1}{4}\left|x_{1}-x_{2}\right|<\frac{1}{2}\left|x_{1}-x_{2}\right| .
$$

This is (1.2) and the theorem follows.

3.9. Remark. F. John proved an injectivity theorem of type 3.8 for bounded convex domains which are uniform by 2.13 (c). However, his method was different from ours.

3.10. Quasiregular mappings in $R^{n}, n \geqq 3$. A mapping $f: G \rightarrow R^{n}, G \subset R^{n}$ a domain, $n \geqq 2$, is called $K$-quasiregular if $f$ is $\mathrm{ACL}^{n}$ and

$$
\left|f^{\prime}(x)\right|^{n} \leqq K J(x, f)
$$


for a.e. $x$ in $G$. The smallest $K$ for which (3.11) is true is denoted by $K(f)$. For the theory of quasiregular mappings in $R^{n}, n \geqq 3$, we refer to [MRV 1-2].

As a counterpart of Theorem 3.7 we prove (see also [ $R$, Lemma 3])

3.12. Theorem. Let $f: B^{n}\left(x_{0}, r\right) \rightarrow R^{n}, n \geqq 3$, be a non-constant $K$-quasiregular mapping. There is a function $\lambda_{n}:[1, \infty) \rightarrow[0, \infty]$ and $q_{n} \in(0,1)$ both depending only on $n$ such that

$$
\lambda_{n}(t) \backslash 0 \text { as } t \rightarrow 1, \text { and }
$$

(3.14) there is $T \in G M(n)$, depending on $f$, such that

for $\left|x-x_{0}\right| \leqq q_{n} r$.

$$
|T \circ f(x)-x| \leqq r \lambda_{n}(K)
$$

Proof. By [MRV 2, Theorems 2.3 and 4.6] there are $K_{0}>1$ and $\varrho \in(0,1)$, both depending only on $n$, such that any non-constant $K_{0}$-quasiregular mapping $f: B^{n} \rightarrow R^{n}$ is injective in $B^{n}(\varrho)$. Let $q=q_{n}=\varrho / 2$. For such $f$ define

and let

$$
\lambda(f)=2 \inf \left\{\sup _{|x| \leqq q}|T \circ f(x)-x|: T \in G M(n)\right\}
$$

$$
\lambda_{n}(K)=\left\{\begin{array}{lll}
\infty, & \text { if } \quad K>K_{0}, \\
\sup \{\lambda(f): K(f) \leqq K\}, & \text { if } \quad 1 \leqq K \leqq K_{0} .
\end{array}\right.
$$

Clearly $\lambda_{n}\left(K_{1}\right) \leqq \lambda_{n}\left(K_{2}\right)$ if $K_{1} \leqq K_{2}$ and by Liouville's theorem in $R^{n}, n \geqq 3, \lambda_{n}(1)=0$. We claim that

$$
\lim _{K \searrow 1} \lambda_{n}(K)=0
$$

Suppose that the limit in (3.15) is $\lambda_{0}>0$. Then we can choose a sequence $f_{i}: B^{n}(2 q) \rightarrow R^{n}, i=1,2, \ldots$, of non-constant $K_{i}$-qc mappings such that

$$
K_{i} \backslash 1 \text { and } \lambda\left(f_{i}\right) \geqq \lambda_{0} / 2>0 \text { for all } i .
$$

Choose Möbius transformations in such a way that $T_{i} \circ f_{i}(y)=y$ for $y \in\{0, q e / 2, q e\}$ and for all $i$ where $e \in S^{n-1}$ is fixed. By a normal family argument (see [V, Corollary 19.5 and Theorem 37.2]) we may assume that the sequence $T_{i} \circ f_{i}$ converges uniformly in $\bar{B}^{n}(q)$ to a Möbius transformation $T$. Then $T(y)=y$ for $y \in\{0, q e / 2, q e\}$ and hence $T$ is a rotation keeping the axis $\{t e: t \in R\}$ fixed. This implies

$$
\begin{aligned}
\frac{1}{2} \lambda\left(f_{i}\right) & \leqq \sup _{|x| \leqq q}\left|T^{-1} \circ T_{i} \circ f_{i}(x)-x\right|=\sup _{|x| \leqq q}\left|T\left(T^{-1} \circ T_{i} \circ f_{i}(x)-x\right)\right| \\
& =\sup _{|x| \leqq q}\left|T_{i} \circ f_{i}(x)-T(x)\right| \rightarrow 0 \quad \text { as } i \rightarrow \infty
\end{aligned}
$$

contradicting (3.16). This proves (3.15).

Now $\lambda_{n}$ defined above satisfies (3.13). To prove (3.14) let $f: B^{n}\left(x_{0}, r\right) \rightarrow R^{n}$ be as in the theorem. Put $g(x)=f\left(x_{0}+r x\right)$ for $x \in B^{n}$. Then by the definition of 
$\lambda_{n}(K)$ there is $T_{1} \in G M(n)$ such that $\left|T_{1} \circ g(x)-x\right| \leqq \lambda_{n}(K)$ for $|x| \leqq q$. Now for $|x| \leqq q$ and $T(y)=r T_{1}(y)+x_{0}$ we get

$$
\begin{aligned}
(1 / r)\left|T \circ f\left(x_{0}+r x\right)-\left(x_{0}+r x\right)\right| & =\left|T_{1} \circ f\left(x_{0}+r x\right)+(1 / r) x_{0}-(1 / r)\left(x_{0}+r x\right)\right| \\
& =\left|T_{1} \circ g(x)-x\right| \leqq \lambda_{n}(K) .
\end{aligned}
$$

This implies the inequality in (3.14).

As in Theorem 3.8 letting $\mathscr{M}=G M(n)$ Theorems 3.3, 3.12 and Proposition 1.1 yield

3.17. Theorem. Let $G$ be an $(\alpha, \beta)$-uniform domain in $R^{n}, n \geqq 3$. There is a constant $c>0$ depending only on $n, \alpha$ and $\beta$ such that $f$ is injective in $G$ whenever $f: G \rightarrow R^{n}$ is $K$-quasiregular and non-constant with $1 \leqq K \leqq 1+c$.

3.18. Remarks. (a) In [S] Theorem 3.17 was proved, by a different method, in the case $G=B^{n}\left(x_{0}, r\right), n \geqq 3$.

(b) For $R^{2}$ Nehari [N] proved: if $f: B^{2} \rightarrow R^{2}$ is analytic and $\sup _{|z|<1}\left|S_{f}(z)\left(1-|z|^{2}\right)^{2}\right| \leqq$ 2 , then $f$ is injective. L. Ahlfors in [A] generalized this as follows: if $f: G \rightarrow R^{2}$ is analytic and $\partial G$ is a qc circle, then there is $\delta>0$ such that $f$ is injective in $D$ whenever $\left\|S_{f}\right\|=\sup _{z \in G}\left|S_{f}(z)\right|$ dist $(z, \partial D)^{2} \leqq \delta$. Now the result in [S] and Theorem 3.17 can be regarded as extension of the theorems of Nehari and Ahlfors, respectively, to $R^{n}, n \geqq 3$, since the Schwarzian norm can be replaced in any domain $G \subset R^{n}$, $n \geqq 3$, by $\log K(f)$ for a non-constant quasiregular mapping $f: G \rightarrow R^{n}$. Observe that $\log K(f)$ and $\left\|S_{f}\right\|$ enjoy the same fundamental property: they vanish if and only if $f=T \mid G$ for some $T \in G M(n)$.

\section{Injectivity theorems in plane}

4.1. In this chapter we apply Proposition 1.1 to plane analytic mappings to get injectivity theorems similar to Theorem 3.17. Instead of the dilatation $K(f)$ we use $f^{\prime \prime} \mid f^{\prime}$ and the Schwarzian derivative $S_{f}=\left(f^{\prime \prime} \mid f^{\prime}\right)^{\prime}-(1 / 2)\left(f^{\prime \prime} \mid f^{\prime}\right)^{2}$ as distortion measures. For them the existence of an approximation of type (1.2) can be proved directly without Rešetnjak's Theorem 3.3, and this leads to injectivity results quantitatively better than the application of 3.3 .

4.2. Let $f: D \rightarrow C$ be analytic in an open set $D \subset C, z_{0} \in D$ and $T$ an analytic function in a neighborhood of $f\left(z_{0}\right)$. Consider the following series expansion:

$$
T \circ f(z)-z=a_{0}+a_{1}\left(z-z_{0}\right)+a_{2}\left(z-z_{0}\right)^{2}+\ldots .
$$

If the first coefficients $a_{0}, a_{1}, \ldots, a_{k}$ vanish, we may consider $T$ a local approximative inverse mapping of $f$ at $z_{0}$. The approximation is the better the larger $k$ is. Especially, we may look for the best possible approximation $T$ in a given function class $\mathscr{M}$. Three classes are of special interest here: $\mathscr{M}_{0}=\{T \in G M(2): T$ is translation $\}$, 
$\mathscr{M}_{1}=\{T \in G M(2): T$ is affine $\}$ and $\mathscr{M}_{2}=G M(2)$. It is easy to see that the best approximation $T \in \mathscr{M}_{0}$ exists and is uniquely determined by the condition $a_{0}=0$ in (4.3), and for this $T$ we have $a_{1}=f^{\prime}\left(z_{0}\right)-1$. The following lemma shows that also in the classes $\mathscr{M}_{k}, k=1,2$, the best approximation exists and it is uniquely determined by the conditions $a_{i}=0,0 \leqq i \leqq k$. Here $f^{\prime \prime}\left(z_{0}\right) / f^{\prime}\left(z_{0}\right)$ and $S_{f}\left(z_{0}\right)$ appear as $(k+1) ! a_{k+1}$, $k=1,2$, respectively.

4.4. Lemma. Let $f: D \rightarrow C$ be analytic, $z_{0} \in D$ and $f^{\prime}\left(z_{0}\right) \neq 0$.

(i) There is a unique $T \in \mathscr{M}_{1}$ such that $T \circ f\left(z_{0}\right)=z_{0}$ and $(T \circ f)^{\prime}\left(z_{0}\right)=1$. For this $T$ we have $(T \circ f)^{\prime \prime}\left(z_{0}\right)=f^{\prime \prime}\left(z_{0}\right) / f^{\prime}\left(z_{0}\right)$.

(ii) There is a unique $T \in \mathscr{M}_{2}$ such that $T \circ f\left(z_{0}\right)=z_{0},(T \circ f)^{\prime}\left(z_{0}\right)=1$ and $(T \circ f)^{\prime \prime}\left(z_{0}\right)=0$. For this $T$ we have $(T \circ f)^{\prime \prime \prime}\left(z_{0}\right)=S_{f}\left(z_{0}\right)$.

Proof. The proofs are easy to obtain by the Taylor expansion of $f$ at $z_{0}$. We consider only the case (ii). We may suppose that $f\left(z_{0}\right)=0$ and $z_{0}=0$. Let $a, b, c, d \in C$, $a d-b c \neq 0$, and consider the Möbius transformation $T(z)=(a z+b) /(c z+d)$. Because $f(0)=0$ and $(T \circ f)(0)$ should vanish, $b=0$ and $d \neq 0$. We may suppose $d=1$. Substitute the expansion

$$
f(z)=f^{\prime}(0) z+\frac{1}{2} f^{\prime \prime}(0) z^{2}+\frac{1}{6} f^{\prime \prime \prime}(0) z^{3}+\left[z^{4}\right]
$$

into the expression $T \circ f(z)-z=a f(z)(c f(z)+1)^{-1}-z$, and observe that it is of the form $a_{3} z^{3}+a_{4} z^{4}+\ldots$ if and only if $a=1 / f^{\prime}(0)$ and $c=(1 / 2) f^{\prime \prime}(0) / f^{\prime}(0)^{2}$, which coefficients yield

$$
a_{3}=\frac{1}{6}\left(\frac{f^{\prime \prime \prime}(0)}{f^{\prime}(0)}-\frac{3}{2}\left(\frac{f^{\prime \prime}(0)}{f^{\prime}(0)}\right)^{2}\right)=\frac{1}{6} S_{f}(0) .
$$

The lemma is proved.

4.5. Remark. For the Schwarzian derivative the above lemma gives an alternative characterization which emphasizes the connection to the approximation of (4.3) with $a_{i}=0, i=0,1,2$. Note that from this characterization one easily obtains the basic relations: $S_{T \circ f}=S_{f}$ and $S_{T}=0$ for all $T \in G M(2)$.

4.6. If $f: D \rightarrow C$ is analytic and injective in a domain $D \subset C$, then

$$
\begin{gathered}
\left|f^{\prime \prime}(z)\right| f^{\prime}(z) \mid \operatorname{dist}(z, \partial D) \leqq 4 \text { and } \\
\left|S_{f}(z)\right| \operatorname{dist}(z, \partial D)^{2} \leqq 6
\end{gathered}
$$

for all $z \in D$, and the bounds are the best possible. For (4.8) see [G, Cor. 1]. To prove (4.7) let $z \in D$ with $r=\operatorname{dist}(z, \partial D)<\infty$. Define $g(w)=[f(z+r w)-f(z)] /\left[r f^{\prime}(z)\right]$, $|w|<1$, and write $g(w)=w+a_{2} w^{2}+a_{3} w^{3}+\ldots$. Because $g$ is univalent in $B^{2}$, we have $2 \geqq\left|a_{2}\right|=(1 / 2) r\left|f^{\prime \prime}(z) / f^{\prime}(z)\right|$, and (4.7) follows. The function $z /(1+z)^{2},|z|<1$, shows that 4 is the best possible in (4.7). These relations suggest the use of the left sides of (4.7) and (4.8) as distortion measures rather than $\left|f^{\prime \prime}\right| f^{\prime} \mid$ and $\left|S_{f}\right|$ in 
the following discussion. The following Theorems 4.9 and 4.14 show that in John domains the local approximation results of Lemma 4.4 can be made global as in Rešetnjak's Theorem 3.3.

4.9. Theorem. Let $D \subset C$ be an $(\alpha, \beta)$-John domain, $0<\alpha \leqq \beta<\infty, f: D \rightarrow C$ analytic, $f^{\prime}(z) \neq 0$ for all $z \in D$ and

$$
\left|\frac{f^{\prime \prime}(z)}{f^{\prime}(z)} \operatorname{dist}(z, \partial D)\right| \leqq k<\frac{\alpha}{\beta} \text { for all } z \in D \text {. }
$$

Let $z_{0} \in D$ be the center of $D$ and $T(z)=a+b z$ for $z \in C$ with $a=z_{0}-f\left(z_{0}\right) / f^{\prime}\left(z_{0}\right)$ and $b=1 / f^{\prime}\left(z_{0}\right)$, i.e. $T \circ f\left(z_{0}\right)=z_{0}$ and $(T \circ f)^{\prime}\left(z_{0}\right)=1$. Then

$$
|T \circ f(z)-z| \leqq \frac{k(\beta / \alpha)}{1-k(\beta / \alpha)} \beta \text { for all } z \in D .
$$

Proof. Let $z \in D, z \neq z_{0}$. Let $\gamma_{1}:\left[0, d_{1}\right] \rightarrow D$ be a rectifiable path with arc length as parameter and such that $\gamma_{1}(0)=z_{0}, \gamma_{1}\left(d_{1}\right)=z, d_{1} \leqq \beta$ and

$$
\operatorname{dist}\left(\gamma_{1}(t), \partial D\right) \geqq \frac{\alpha}{\beta}\left(d_{1}-t\right) \text { for } 0 \leqq t \leqq d_{1} .
$$

Let $k \beta<\alpha^{\prime}<\alpha$. It is not difficult to see that we can replace $\gamma_{1}$ by a rectifiable Jordan arc $\gamma:[0, d] \rightarrow D$ with arc length as parameter and such that $\gamma$ is (finitely) piecewise affine, $\gamma(0)=z_{0}, \gamma(d)=z, d \leqq d_{1}$, and

$$
\operatorname{dist}(\gamma(t), \partial D) \geqq \frac{\alpha^{\prime}}{\beta}(d-t) \text { for } 0 \leqq t \leqq d .
$$

Then it is easy to choose a simply connected domain $G \subset D$ in such a way that $\gamma[0, d] \subset G$. Observe that the function

$$
\varphi(u)=\int_{z_{0}}^{u} \exp \left(\int_{z_{0}}^{v} \frac{f^{\prime \prime}(w)}{f^{\prime}(w)} d w\right) d v+z_{0}, \quad u \in G,
$$

is a solution of the equation

$$
y^{\prime \prime}(u)-\frac{f^{\prime \prime}(u)}{f^{\prime}(u)} y^{\prime}(u)=0, \quad u \in G
$$

with $\varphi\left(z_{0}\right)=z_{0}$ and $\varphi^{\prime}\left(z_{0}\right)=1$. Because $T \circ f$ is also a solution of (4.12) with the same initial values at $z_{0}$, we have, by uniqueness, $T \circ f(u)=\varphi(u)$ for $u \in G$. Set

We obtain

$$
h(u)=\int_{z_{0}}^{u} \frac{f^{\prime \prime}(w)}{f^{\prime}(w)} d w, \quad u \in G .
$$

$$
\begin{aligned}
|T \circ f(z)-z| & =\left|\int_{0}^{d}[\exp (h \circ \gamma(t))-1] \gamma^{\prime}(t) d t\right| \\
& \leqq \int_{0}^{d}[\exp (|h \circ \gamma(t)|)-1] d t .
\end{aligned}
$$


On the other hand, by (4.10) and (4.11)

$$
\begin{aligned}
|h(\gamma(t))| & =\left|\int_{0}^{t} \frac{f^{\prime \prime} \circ \gamma(s)}{f^{\prime} \circ \gamma(s)} \gamma^{\prime}(s) d s\right| \leqq \int_{0}^{t}\left|\frac{f^{\prime \prime} \circ \gamma(s)}{f^{\prime} \circ \gamma(s)}\right| d s \\
& \leqq \int_{0}^{t} k \frac{\beta}{\alpha^{\prime}}(d-s)^{-1} d s=\log \left[\left(\frac{d}{d-t}\right)^{k^{\prime}}\right],
\end{aligned}
$$

where $k^{\prime}=k \beta / \alpha^{\prime}$. Substitute this into (4.13) and get

$$
|T \circ f(z)-z| \leqq \frac{1}{1-k^{\prime}} d-d \leqq \frac{k^{\prime}}{1-k^{\prime}} \beta .
$$

Finally let $\alpha^{\prime} \rightarrow \alpha$ and the theorem follows.

4.14. Theorem. Let $D \subset C$ be an $(\alpha, \beta)$-John domain, $0<\alpha \leqq \beta<\infty, f: D \rightarrow C$ analytic, $f^{\prime}(z) \neq 0$ for all $z \in D$ and

$$
2\left|S_{f}(z)\right| \operatorname{dist}(z, \partial D)^{2} \leqq k<\left(\frac{\alpha}{\beta}\right)^{2} \text { for all } z \in D
$$

Let $z_{0} \in D$ be the John center of $D$ and $T$ the unique Möbius transformation with $T \circ f\left(z_{0}\right)=z_{0},(T \circ f)^{\prime}\left(z_{0}\right)=1$ and $(T \circ f)^{\prime \prime}\left(z_{0}\right)=0$. Then

$$
|T \circ f(z)-z| \leqq \frac{k(\beta / \alpha)^{2}}{1-k(\beta / \alpha)^{2}} \beta \text { for all } z \in D .
$$

Proof. Let $z \in D, z \neq z_{0}$. Let $\gamma:[0, d] \rightarrow D$ be a rectifiable path with arc length as parameter such that $\gamma(0)=z_{0}, \gamma(d)=z, d \leqq \beta$ and

$$
\operatorname{dist}(\gamma(t), \partial D) \geqq \frac{\alpha}{\beta}(d-t) \text { for } \quad 0 \leqq t \leqq d .
$$

The reasoning in the proof of Theorem 4.9 shows that we may assume $\gamma$ to be a piecewise affine Jordan arc.

Let $g=T \circ f: D \rightarrow \bar{C}$. Then $g$ is meromorphic and

$$
g\left(z_{0}\right)=z_{0}, \quad g^{\prime}\left(z_{0}\right)=1 \text { and } g^{\prime \prime}\left(z_{0}\right)=0 .
$$

Furthermore, $S_{f}(z)=S_{g}(z)=\left(g^{\prime \prime} / g^{\prime}\right)^{\prime}-(1 / 2)\left(g^{\prime \prime} / g^{\prime}\right)^{2}$, and so

(4.19) $\quad w^{\prime}(z)=S_{f}(z)+\frac{1}{2} w(z)^{2}, \quad w\left(z_{0}\right)=0, \quad$ where $\quad w(z)=\frac{g^{\prime \prime}(z)}{g^{\prime}(z)}, \quad z \in D$.

We show that

$$
|w(\gamma(t))| \leqq k \frac{\beta^{2}}{\alpha^{2}} \frac{1}{d-t} \quad \text { for } \quad 0 \leqq t<d,
$$

after which we can proceed as in Theorem 4.9. To prove (4.20) begin with observing that $\varphi(t)=k(\beta / \alpha)^{2}(d-t)^{-1}, 0 \leqq t<d$, satisfies the differential equation

$$
\varphi^{\prime}(t)=\chi\left(t,+\frac{1}{2} \varphi(t)^{2}, \quad 0 \leqq t<d \quad \text { and } \quad \varphi(0)=\frac{k \beta^{2}}{\alpha^{2} d},\right.
$$


where $x(t)=\left(k(\beta / \alpha)^{2}-(1 / 2)\left(k \beta^{2} / \alpha^{2}\right)^{2}\right)(d-t)^{-2}$. Let

$$
\omega(t)=|w(\gamma(t))|+\frac{k \beta^{2}}{\alpha^{2} d} \quad \text { for } \quad 0 \leqq t<d .
$$

Let $S=\{s \in(0, d): w(\gamma(t))$ bounded for $t \in[0, s]\}$ and $t_{1}=\sup S$. Note that $S \neq \emptyset$ by (4.18). Because $w$ is meromorphic we only need to prove (4.20) for $t \in\left[0, t_{1}\right)$ since then we have $t_{1}=d$.

Now $\omega:\left[0, t_{1}\right) \rightarrow \boldsymbol{R}$ is absolutely continuous on closed subintervals of $\left[0, t_{1}\right)$. We get for almost every $t \in\left[0, t_{1}\right)$ by (4.19), (4.15) and (4.17)

$$
\begin{gathered}
\omega^{\prime}(t) \leqq\left|w^{\prime}(\gamma(t)) \gamma^{\prime}(t)\right|=\left|w^{\prime}(\gamma(t))\right|=\left|S_{f}(\gamma(t))+\frac{1}{2} w(\gamma(t))^{2}\right| \\
\leqq\left|S_{f}(\gamma(t))\right|+\frac{1}{2} \omega(t)^{2} \leqq \frac{1}{2} k\left(\frac{\beta}{\alpha}\right)^{2}(d-t)^{-2}+\frac{1}{2} \omega(t)^{2} \leqq \chi(t)+\frac{1}{2} \omega(t)^{2} .
\end{gathered}
$$

Next, define for $t \in\left[0, t_{1}\right)$

$$
\psi(t)=\frac{k \beta^{2}}{\alpha^{2} d}+\int_{0}^{t}\left[\varkappa(s)+\frac{1}{2} \omega(s)^{2}\right] d s \geqq \frac{k \beta^{2}}{\alpha^{2} d}+\int_{0}^{t} \omega^{\prime}(s) d s=\omega(t) .
$$

Then $\psi:\left[0, t_{1}\right) \rightarrow \boldsymbol{R}$ is continuously differentiable and

$$
\psi^{\prime}(t)=\varkappa(t)+\frac{1}{2} \omega(t)^{2} \leqq \chi(t)+\frac{1}{2} \psi(t)^{2} \quad \text { for } \quad 0 \leqq t<t_{1}
$$

and $\psi(0)=k \beta^{2} / \alpha^{2} d$. Then a standard differential inequality reasoning (see e.g. [BR, p. 22]) yields

$$
|w(\gamma(t))| \leqq \omega(t) \leqq \psi(t) \leqq \varphi(t) \text { for } \quad t \in\left[0, t_{1}\right) .
$$

This proves (4.20).

Let $s \in(0, d)$ be arbitrary. Because $\gamma$ is a piecewise linear Jordan arc, we can choose a simply connected domain $G \subset D$ in such a way that $\gamma[0, s] \subset G$. By (4.20) $w=g^{\prime \prime} \mid g^{\prime}$ is analytic on $\gamma[0, s]$, and therefore we may assume that $g^{\prime \prime} \mid g^{\prime}$ is analytic in the entire $G$. Now, $g$ is in $G$ the unique solution of the equation

$$
y^{\prime \prime}-\frac{g^{\prime \prime}}{g^{\prime}} y^{\prime}=0
$$

with $g\left(z_{0}\right)=z_{0}$ and $g^{\prime}\left(z_{0}\right)=1$ by (4.18). This implies, by uniqueness,

Write

$$
g(v)=\int_{z_{0}}^{v} \exp \left(\int_{z_{0}}^{u} \frac{g^{\prime \prime}(t)}{g^{\prime}(t)} d t\right) d u+z_{0}, \quad v \in G .
$$

$$
h(u)=\int_{z_{0}}^{u} \frac{g^{\prime \prime}(t)}{g^{\prime}(t)} d t, \quad u \in G .
$$


Then due to (4.20) we get

$$
|h(\gamma(t))|=\left|\int_{0}^{t} w(\gamma(r)) \gamma^{\prime}(r) d r\right| \leqq \int_{0}^{t} k \frac{\beta^{2}}{\alpha^{2}} \frac{1}{d-r} d r=\log \left[\left(\frac{d}{d-t}\right)^{k^{\prime}}\right], \quad 0 \leqq t \leqq s,
$$

where $k^{\prime}=k \beta^{2} / \alpha^{2}$. Furthermore, we get, as in Theorem 4.9,

$$
\begin{gathered}
|g(\gamma(s))-\gamma(s)|=\left|\int_{0}^{s}[\exp (h(\gamma(t)))-1] \gamma^{\prime}(t) d t\right| \\
\leqq \int_{0}^{s}\left[\left(\frac{d}{d-t}\right)^{k^{\prime}}-1\right] d t=\frac{1}{1-k^{\prime}}\left(d-d^{k^{\prime}}(d-s)^{1-k^{\prime}}\right)-s \leqq \frac{1}{1-k^{\prime}} d-s .
\end{gathered}
$$

Let $s \rightarrow d$ and, because $g=T \circ f$ is meromorphic, (4.16) follows from (4.23). The theorem is proved.

4.24. Theorem. Let $f: D \rightarrow C$ be analytic in an $(\alpha, \beta)$-uniform domain $D \subset C$, $0<\alpha \leqq \beta<\infty$, and $f^{\prime}(z) \neq 0$ for all $z \in D$. If either

$$
\sup _{z \in D} \frac{\left|f^{\prime \prime}(z)\right|}{\left|f^{\prime}(z)\right|} \operatorname{dist}(z, \partial D)<\frac{\alpha}{\beta}(2 \beta+1)^{-1},
$$

or

$$
\sup _{z \in D}\left|S_{f}(z)\right| \operatorname{dist}(z, \partial D)^{2}<\frac{1}{2}\left(\frac{\alpha}{\beta}\right)^{2}(2 \beta+1)^{-1},
$$

then $f$ is injective in $D$.

Proof. Observe that if $z \in G \subset D$, then $\operatorname{dist}(z, \partial G) \leqq \operatorname{dist}(z, \partial D)$. The proof follows now from Theorems 4.9 and 4.14 and Proposition (1.1).

4.25. Remark. Let $D$ be the unit disk and $f: D \rightarrow C$ analytic with $f^{\prime}(z) \neq 0$ for $z \in D$. By Becker [B] and Nehari $[\mathrm{N}] f$ is injective if $\sup _{z \in D}\left|f^{\prime \prime}(z) / f^{\prime}(z)\right|(1-|z|)<$ $1 / 2$ or $\sup _{z \in D}\left|S_{f}(z)\right|(1-|z|)^{2}<1 / 2$. We can compare this to the above theorem. By 2.13 (a) $D \in U(1 / 2, \sqrt{2} / 2)$ and for these $\alpha$ and $\beta$ (they are not the best possible) we get $(\alpha / \beta)(2 \beta+1)^{-1}=0,292 \ldots$ and $(1 / 2)(\alpha / \beta)^{2}(2 \beta+1)^{-1}=0,103 \ldots$. The above theorem also extends Ahlfors' theorem [A], because a plane domain whose boundary is a $K$-qc circle is $(\alpha, \beta)$-uniform with $\alpha$ and $\beta$ depending only on $K$; see Theorem 2.15 . Observe that the proof of 4.24 (ii) is different from that of [N]. Especially, we did not use the well-known fact that if $S_{f}=2 Q$, then $f=y_{1} / y_{2}$, where $y_{i}, i=1,2$, are solutions of $y^{\prime \prime}+Q y=0$.

4.26. Theorem 4.24 can be improved if it assumed that $\left|f^{\prime \prime}\right| f^{\prime} \mid$ and $\left|S_{f}\right|$ are uniformly small in the domain $D$. This leads us to consider quasiconvex domains. We say that a domain $D \subset R^{n}, n \geqq 2$, is $\sigma$-quasiconvex, $\sigma \geqq 1$, if any $x, y \in D$ can be joined in $D$ by a rectifiable path whose length does not exceed $\sigma|x-y|$. Note that an $(\alpha, \beta)$-uniform domain is $2 \beta$-quasiconvex. 
4.27. Theorem. Let $D \subset C$ be a $\sigma$-quasiconvex domain, $\operatorname{diam}(D)=d<\infty$, and $f: D \rightarrow C$ analytic with $f^{\prime}(z) \neq 0$ for all $z \in D$.

(i) If $\sup _{z \in D}\left|f^{\prime \prime}(z) / f^{\prime}(z)\right|=k<\varkappa_{1} /\left(d \sigma^{2}\right)$, then $f$ is injective. Here $x_{1}=1,256 \ldots$ satisfies $e^{x_{1}}=1+2 x_{1}$.

(ii) If $\sup _{z \in D}\left|S_{f}(z)\right|=k<2 \varkappa_{2}^{2} /\left(d^{2} \sigma^{3}\right)$, then $f$ is injective. Here $\varkappa_{2}=1.165 \ldots$ satisfies $2 x_{2}=\tan \varkappa_{2}$.

Proof. Suppose that $f\left(z_{1}\right)=f\left(z_{2}\right)$ for some $z_{1}, z_{2} \in D, z_{1} \neq z_{2}$. Let $G \subset D$ be a simply connected domain and $\gamma:[0, l] \rightarrow G$ a rectifiable curve, arc length as parameter, such that $\gamma(0)=z_{1}, \gamma(l)=z_{2}$ and $l \leqq \sigma r, r=\left|z_{2}-z_{1}\right|>0$.

To prove (i) let $T \in G M(2)$ be affine such that $T \circ f\left(z_{1}\right)=z_{1}$ and $(T \circ f)^{\prime}\left(z_{1}\right)=1$ By reasoning as in Theorem 4.9 we get

$$
\begin{aligned}
r & =\left|z_{2}-z_{1}\right|=\left|T \circ f\left(z_{2}\right)-z_{1}-\left(z_{2}-z_{1}\right)\right|=\left|\int_{z_{1}}^{z_{2}}\left[\exp \left(\int_{z_{1}}^{u} \frac{f^{\prime \prime}(v)}{f^{\prime}(v)} d v\right)-1\right] d u\right| \\
& \leqq \int_{0}^{l}\left[e^{k t}-1\right] d t=\frac{1}{k}\left(e^{k l}-1\right)-l \leqq \sigma^{2} r^{2} k \varphi(k \sigma d),
\end{aligned}
$$

where $\varphi(x)=x^{-2}\left(e^{x}-1-x\right), x>0$, which implies

$$
\frac{1}{\sigma^{2} d} \leqq k \varphi(k \sigma d)
$$

Note that $\varphi\left(\varkappa_{1}\right)=\chi_{1}^{-1}$. By assumption $k<\varkappa_{1} /\left(d \sigma^{2}\right)$, which yields $k \sigma d<\chi_{1} \sigma^{-1} \leqq$ $\varkappa_{1}$. Therefore, $k x_{1}^{-1}=k \varphi\left(x_{1}\right)>k \varphi(k \sigma d) \geqq 1 /\left(\sigma^{2} d\right)$ by (4.28). This is a contradiction.

To prove (ii) let $T \in G M(2)$ such that $g\left(z_{1}\right)=z_{1}, g^{\prime}\left(z_{1}\right)=1$ and $g^{\prime \prime}\left(z_{1}\right)=0$ for $g=T \circ f$. Observe that $\varphi(t)=a \tan (a t / 2)$ satisfies $\varphi(0)=0$ and $\varphi^{\prime}(t)=k+(1 / 2) \varphi(t)^{2}$ for $0 \leqq t a<\pi$ and $a=(2 k)^{1 / 2}$. By reasoning as in Theorem 4.14 we get

$$
\begin{aligned}
r= & \left|z_{1}-z_{2}\right|=\left|g\left(z_{2}\right)-z_{1}-\left(z_{2}-z_{1}\right)\right|=\left|\int_{z_{1}}^{z_{2}}\left[\exp \left(\int_{z_{1}}^{u} \frac{g^{\prime \prime}(v)}{g^{\prime}(v)} d v\right)-1\right] d u\right| \\
& \leqq \int_{0}^{i}\left[\exp \left(\int_{0}^{u} a \tan \left(\frac{t a}{2}\right) d t\right)-1\right] d u=\frac{2}{a} \tan \left(\frac{l a}{2}\right)-l= \\
& =\alpha^{2} l^{3} \psi(\alpha l) \leqq \alpha^{2} \sigma^{3} r^{3} \psi(\alpha \sigma d),
\end{aligned}
$$

where $\alpha=a / 2$ and $\psi(x)=x^{-3}(\tan x-x)$ for $x>0$. This implies

$$
\frac{1}{\sigma^{3} d^{2}} \leqq \alpha^{2} \psi(d \sigma \alpha)
$$

Note that $\psi\left(\varkappa_{2}\right)=x_{2}^{-2}$. By assumption $k<2 x_{2}^{2} d^{-2} \sigma^{-3}$, which implies $\alpha \sigma d=$ $(k / 2)^{1 / 2} \sigma d<\varkappa_{2} \sigma^{-1 / 2} \leqq \varkappa_{2}$. With (4.29) this yields $\sigma^{-3} d^{-2} \leqq \alpha^{2} \psi(d \sigma \alpha)<\alpha^{2} \psi\left(\varkappa_{2}\right)=$ $k /\left(2 x_{2}^{2}\right)$, which is a contradiction. The proof is complete. 


\section{References}

[A] Ahlfors, L.: Quasiconformal reflections. - Acta Math. 109, 1963, 291-301.

[B] BeCKeR, J.: Über homomorphe Fortsetzung schlichter Funktionen. - Ann. Acad. Sci. Fenn. Ser. A I 538, 1973, 1-11.

[BR] Birkhoff, G., and G-C. Rota: Ordinary differential equations. - Blaisdell Publishing Company, 1962.

[DSS] Duren, P., H. Shapiro, and A. Shields: Singular measures and domains not of Smirnov type. - Duke Math. J. 33, 1963, 245-254.

[G] Gehring, F.: Univalent functions and the Schwarzian derivative. - Comment. Math. Helv. 52, 1977, 561-572.

[GV] GeHrING, F., and J. VÄISÄLÄ: The coefficients of quasiconformality of domains in space. Acta Math. 114, 1965, 1-70.

[H] Hille, E.: Lectures on ordinary differential equations. - Addison-Wesley Publishing Company, Reading, Massachusetts-Menlo Park, California-London-Don Mills, Ontario, 1969.

[J] JohN, F.: Rotation and strain. - Comm. Pure Appl. Math. 14, 1961, 391-413.

[MRV 1] MARTIO, O., S. RICKMAN, and J. VÄISÄLÄ: Definitions for quasiregular mappings. - Ann. Acad. Sci. Fenn. Ser. A I 448, 1969, $1-40$.

[MRV 2] MARTIO, O., S. R.ICKMAN, and J. VÄISÄLÄ: Topological and metric properties of quasiregular mappings. - Ibid. 488, 1971, 1-31.

[N] Nehari, Z.: The Schwarzian derivative and schlicht functions. - Bull. Amer. Math. Soc. $55,1949,545-551$.

[Ne] Newman, M. H. A.: Elements of the topology of plane sets of points. - Cambridge Univ. Press, Cambridge, 1954.

[R] REŠETNJAK, JU. G.: Stability in Liouville's theorem on conformal mappings of a space for domains with nonsmooth boundary. - Sibirsk. Mat. Ž. 17, 1976, 361-369 (Russian).

[S] Sarvas, J.: Coefficient of injectivity for quasiregular mappings. - Duke Math. J. 43, 1976, 147-158.

[V] VÄISÄLÄ, J.: Lectures on $n$-dimensional quasiconformal mappings. - Lecture Notes in Mathematics 229, Springer-Verlag, Ferlin-Heidelberg-New York, 1971.

University of Helsinki

Department of Mathematics

SF-00100 Helsinki 10

Finland

Received 23 January 1979 